\title{
ABSTRACT CONVOLUTION FUNCTION ALGEBRAS OVER HOMOGENEOUS SPACES OF COMPACT GROUPS
}

\author{
ARASH GHAANI FARASHAHI
}

\begin{abstract}
This paper presents a systematic study for structure of abstract Banach function *-algebras over homogeneous spaces of compact groups. Let $G$ be a compact group and $H$ be a closed subgroup of $G$. Let $\mu$ be the normalized $G$-invariant measure over the homogeneous space $G / H$ associated to the Weil's formula and $1 \leq p<\infty$. Then we introduce the notions of convolution and involution for the Banach function spaces $L^{p}(G / H, \mu)$.
\end{abstract}

\section{Introduction}

The mathematical theory of Banach convolution algebras plays significant and classical roles in abstract harmonic analysis, representation theory, functional analysis, operator theory, and $C^{*}$-algebras, see [1], [2], [3], [11], [12], [13], [15], [16], [22], [23] and references therein. Over the last decades, some new aspects and applications of Banach convolution algebras have achieved significant popularity in time-frequency (Gabor) analysis, coorbit theory, see [4], [5], [6], [7], [8], [14] and references therein.

The following paper introduces the abstract notions of convolution and involution over the functions spaces related to homogeneous spaces (coset spaces) of compact groups. In a nutshell, homogeneous spaces are group-like structures with many interesting applications in mathematical physics, differential geometry, geometric analysis, and coherent state (covariant) transforms, see [10], [17], [18], [19], [20], [21].

This article contains 4 sections. Section 2 is devoted to fix notations and provides a summary of classical harmonic analysis over compact groups and homogeneous spaces (left coset spaces) of compact groups. Let $G$ be a compact group and $H$ be a closed subgroup of $G$. Let $\mu$ be the normalized $G$ invariant measure over the homogeneous space $G / H$ associated to the Weil's

Received April 9, 2016; received in final form July 7, 2016.

2010 Mathematics Subject Classification. 22C05, 43A15, 43A20, 43A77, 43A85, $47 \mathrm{~A} 67$. 
formula and $1 \leq p<\infty$. In Section 3, we study abstract harmonic analysis over the Banach functions spaces related to homogeneous spaces of compact groups. Then we introduce the abstract notion of generalized convolution for $L^{p}$-functions spaces over homogeneous spaces of compact groups. We also study properties of these convolutions. As a result, we shall show that the Banach function space $L^{p}(G / H, \mu)$ is a Banach algebra, with respect to generalized convolutions. Then we introduce the notion of generalized involution for functions over homogeneous spaces of compact groups and we address properties of these involutions, such as behavior of involutions and convolutions on each other. Finally, we show that with respect to these convolutions and involutions, some Banach functions spaces over homogeneous spaces of compact groups are Banach $*$-algebras.

\section{Preliminaries and notations}

Let $X$ be compact Hausdorff space. By $\mathcal{C}(X)$ we mean the space of all continuous complex valued functions on $X$. If $\mu$ is a positive Radon measure on $X$, for each $1 \leq p<\infty$ the Banach space of equivalence classes of $\mu$-measurable complex valued functions $f: X \rightarrow \mathbb{C}$ such that

$$
\|f\|_{L^{p}(X, \mu)}=\left(\int_{X}|f(x)|^{p} d \mu(x)\right)^{1 / p}<\infty,
$$

is denoted by $L^{p}(X, \mu)$ which contains $\mathcal{C}(X)$ as a $\|\cdot\|_{L^{p}(X, \mu)}$-dense subspace.

Let $G$ be a compact group with the probability Haar measure $d x$. For $p \geq 1$ the notation $L^{p}(G)$ stands for the Banach function space $L^{p}(G, d x)$. The standard convolution for $f, g \in L^{1}(G)$, is defined via

$$
f *_{G} g(x)=\int_{G} f(y) g\left(y^{-1} x\right) d y \quad(x \in G) .
$$

The involution for $f \in L^{p}(G)$, is defined by $f^{*^{G}}(x)=\overline{f\left(x^{-1}\right)}$ for $x \in G$. Then the Banach function space $L^{p}(G)$ equipped with the above convolution and involution is a Banach $*$-algebra, that is

$$
\left\|f *_{G} g\right\|_{p} \leq\|f\|_{p}\|g\|_{p},
$$

and

$$
\left(f *_{G} g\right)^{*^{G}}=g^{*^{G}} *_{G} f^{*^{G}},
$$

for all $f, g \in L^{p}(G)$, see [9], [15], [16], [23] and classical list of references therein.

Let $H$ be a closed subgroup of $G$ with the probability Haar measure $d h$. The left coset space $G / H$ is considered as a locally compact homogeneous space that $G$ acts on it from the left, and $q: G \rightarrow G / H$ given by $x \mapsto q(x):=x H$ is the surjective canonical map. The classical aspects of abstract harmonic analysis on locally compact homogeneous spaces are quite well studied by several authors, see [9], [15], [16], [23] and references therein. If $G$ is compact, 
each transitive $G$-space can be considered as a left coset space $G / H$ for some closed subgroup $H$ of $G$. The function space $\mathcal{C}(G / H)$ consists of all functions $T_{H}(f)$, where $f \in \mathcal{C}(G)$ and

$$
T_{H}(f)(x H)=\int_{H} f(x h) d h .
$$

Let $\mu$ be a Radon measure on $G / H$ and $x \in G$. The translation $\mu_{x}$ of $\mu$ is defined by $\mu_{x}(E)=\mu(x E)$, for all Borel subsets $E$ of $G / H$. The measure $\mu$ is called $G$-invariant if $\mu_{x}=\mu$, for all $x \in G$. The homogeneous space $G / H$ has a normalized $G$-invariant measure $\mu$, which satisfies the Weil's formula

$$
\int_{G / H} T_{H}(f)(x H) d \mu(x H)=\int_{G} f(x) d x
$$

and hence the linear map $T_{H}$ is norm-decreasing, that is

$$
\left\|T_{H}(f)\right\|_{L^{1}(G / H, \mu)} \leq\|f\|_{L^{1}(G)}
$$

for all $f \in L^{1}(G)$, see [9], [15], [23].

For a function $\varphi \in L^{p}(G / H, \mu)$ and $z \in G$, the left action of $z$ on $\varphi$ is defined by $L_{z} \varphi(x H)=\varphi\left(z^{-1} x H\right)$ for $x H \in G / H$. Then it can be readily checked that $L_{z}: L^{p}(G / H, \mu) \rightarrow L^{p}(G / H, \mu)$ is a unitary operator.

\section{Abstract harmonic analysis over homogeneous spaces of compact groups}

Throughout this paper, we assume that $G$ is a compact group with the probability Haar measure $d x, H$ is a closed subgroup of $G$ with the probability Haar measure $d h$, and $\mu$ is the normalized $G$-invariant measure on the compact homogeneous space $G / H$ satisfying (4) with respect to the probability Haar measures of $G$ and $H$. From now on, we may say $\mu$ is the normalized $G$ invariant measure over the compact homogeneous space $G / H$, at times.

The following proposition shows that the linear map $T_{H}: \mathcal{C}(G) \rightarrow \mathcal{C}(G / H)$ is uniformly continuous.

Proposition 1. Let $H$ be a closed subgroup of a compact group $G$. The linear map $T_{H}: \mathcal{C}(G) \rightarrow \mathcal{C}(G / H)$ is uniformly continuous.

Proof. Let $f \in \mathcal{C}(G)$ and $x \in G$. Then we have

$$
\left|T_{H}(f)(x H)\right|=\left|\int_{H} f(x h) d h\right| \leq \int_{H}|f(x h)| d h \leq\|f\|_{\text {sup }}\left(\int_{H} d h\right)=\|f\|_{\text {sup }},
$$

which implies $\left\|T_{H}(f)\right\|_{\text {sup }} \leq\|f\|_{\text {sup }}$.

The next theorem proves that the linear map $T_{H}$ is norm-decreasing in other $L^{p}$-spaces, when $p>1$. 
THEOREM 2. Let $H$ be a closed subgroup of a compact group $G, \mu$ be the normalized $G$-invariant measure on $G / H$, and $p \geq 1$. The linear map $T_{H}: \mathcal{C}(G) \rightarrow \mathcal{C}(G / H)$ satisfies

$$
\left\|T_{H}(f)\right\|_{L^{p}(G / H, \mu)} \leq\|f\|_{L^{p}(G)},
$$

for all $f \in \mathcal{C}(G)$. Hence, it has a unique extension to a norm-decreasing linear map from $L^{p}(G)$ onto $L^{p}(G / H, \mu)$.

Proof. Let $f \in \mathcal{C}(G)$. Using compactness of $H$, and the Weil's formula, we can write

$$
\begin{aligned}
\int_{G / H}\left|T_{H}(f)(x H)\right|^{p} d \mu(x H) & =\int_{G / H}\left|\int_{H} f(x h) d h\right|^{p} d \mu(x H) \\
& \leq \int_{G / H}\left(\int_{H}|f(x h)| d h\right)^{p} d \mu(x H) \\
& \leq \int_{G / H} \int_{H}|f(x h)|^{p} d h d \mu(x H) \\
& =\int_{G / H} \int_{H}|f|^{p}(x h) d h d \mu(x H) \\
& =\int_{G / H} T_{H}\left(|f|^{p}\right)(x H) d \mu(x H)=\int_{G}|f(x)|^{p} d x
\end{aligned}
$$

which implies (5). Thus, we can extend $T_{H}$ to a bounded linear operator from $L^{p}(G)$ onto $L^{p}(G / H, \mu)$, which still will be denoted by $T_{H}$ and satisfies (5) for all $f \in L^{p}(G)$.

As an immediate consequence of Theorem 2 we deduce the following corollary.

Corollary 3. Let $H$ be a closed subgroup of a compact group $G, \mu$ be the normalized $G$-invariant measure on $G / H$, and $p \geq 1$. Let $\varphi \in L^{p}(G / H, \mu)$ and $\varphi_{q}:=\varphi \circ q$. Then $\varphi_{q} \in L^{p}(G)$ with

$$
\left\|\varphi_{q}\right\|_{L^{p}(G)}=\|\varphi\|_{L^{p}(G / H, \mu)} .
$$

Proof. Indeed, using the Weil's formula, we can write

$$
\begin{aligned}
\left\|\varphi_{q}\right\|_{L^{p}(G)}^{p} & =\int_{G}\left|\varphi_{q}(x)\right|^{p} d x \\
& =\int_{G / H} T_{H}\left(\left|\varphi_{q}\right|^{p}\right)(x H) d \mu(x H) \\
& =\int_{G / H}\left(\int_{H}\left|\varphi_{q}(x h)\right|^{p} d h\right) d \mu(x H),
\end{aligned}
$$


and since $H$ is compact and $d h$ is normalized, we get

$$
\begin{aligned}
\int_{G / H}\left(\int_{H}\left|\varphi_{q}(x h)\right|^{p} d h\right) d \mu(x H) & =\int_{G / H}\left(\int_{H}|\varphi(x h H)|^{p} d h\right) d \mu(x H) \\
& =\int_{G / H}\left(\int_{H}|\varphi(x H)|^{p} d h\right) d \mu(x H) \\
& =\int_{G / H}|\varphi(x H)|^{p}\left(\int_{H} d h\right) d \mu(x H) \\
& =\int_{G / H}|\varphi(x H)|^{p} d \mu(x H),
\end{aligned}
$$

which implies (6).

The next proposition shows that the linear operator $T_{H}: L^{2}(G) \rightarrow$ $L^{2}(G / H, \mu)$ is a partial isometric linear map.

Proposition 4. Let $H$ be a closed subgroup of a compact group $G$ and $\mu$ be the normalized $G$-invariant measure on $G / H$. Then $T_{H}: L^{2}(G) \rightarrow$ $L^{2}(G / H, \mu)$ is a partial isometric linear map.

Proof. Let $\varphi \in L^{2}(G / H, \mu)$. Invoking Corollary 3 we claim that $T_{H}^{*}(\varphi)=$ $\varphi_{q}$, and hence $T_{H} T_{H}^{*}(\varphi)=\varphi$. Indeed, using the Weil's formula, we can write

$$
\begin{aligned}
\left\langle T_{H}^{*}(\varphi), f\right\rangle_{L^{2}(G)} & =\left\langle\varphi, T_{H}(f)\right\rangle_{L^{2}(G / H, \mu)} \\
& =\int_{G / H} \varphi(x H) \overline{T_{H}(f)(x H)} d \mu(x H) \\
& =\int_{G / H} \varphi(x H) T_{H}(\bar{f})(x H) d \mu(x H) \\
& =\int_{G / H} T_{H}\left(\varphi_{q} \cdot \bar{f}\right)(x H) d \mu(x H) \\
& =\int_{G} \varphi_{q}(x) \overline{f(x)} d x=\left\langle\varphi_{q}, f\right\rangle_{L^{2}(G)},
\end{aligned}
$$

for all $f \in L^{2}(G)$, which implies that $T_{H}^{*}(\varphi)=\varphi_{q}$. Now a straightforward calculation shows that $T_{H}=T_{H} T_{H}^{*} T_{H}$. Then, by Theorem 2.3.3 of [22], $T_{H}$ is a partial isometric operator.

The following corollaries are straightforward consequences of Proposition 4. Let $\mathcal{J}^{2}(G, H):=\left\{f \in L^{2}(G): T_{H}(f)=0\right\}$ and $\mathcal{J}^{2}(G, H)^{\perp}$ be the orthogonal complement of the closed subspace $\mathcal{J}^{2}(G, H)$ in $L^{2}(G)$.

Corollary 5. Let $H$ be a closed subgroup of a compact group G. Let $P_{\mathcal{J}^{2}(G, H)}$ (resp. $\left.P_{\mathcal{J}^{2}(G, H)^{\perp}}\right)$ be the orthogonal projections onto the closed subspaces $\mathcal{J}^{2}(G, H)\left(\right.$ resp. $\left.\mathcal{J}^{2}(G, H)^{\perp}\right)$. Then for each $f \in L^{2}(G)$ and for a.e. 
$x \in G$ we have

$$
\begin{aligned}
P_{\mathcal{J}^{2}(G, H)^{\perp}}(f)(x) & =T_{H}(f)(x H), \\
P_{\mathcal{J}^{2}(G, H)}(f)(x) & =f(x)-T_{H}(f)(x H) .
\end{aligned}
$$

Corollary 6. Let $H$ be a compact subgroup of a locally compact group $G$ and $\mu$ be the normalized $G$-invariant measure on $G / H$. Then

(1) $\mathcal{J}^{2}(G, H)^{\perp}=\left\{\psi_{q}=\psi \circ q: \psi \in L^{2}(G / H, \mu)\right\}$.

(2) For $f \in \mathcal{J}^{2}(G, H)^{\perp}$ and $h \in H$, we have $R_{h} f=f$.

(3) For $\psi \in L^{2}(G / H, \mu)$, we have $\left\|\psi_{q}\right\|_{L^{2}(G)}=\|\psi\|_{L^{2}(G / H, \mu)}$.

(4) For $f, g \in \mathcal{J}^{2}(G, H)^{\perp}$, we have

$$
\left\langle T_{H}(f), T_{H}(g)\right\rangle_{L^{2}(G / H, \mu)}=\langle f, g\rangle_{L^{2}(G)} .
$$

REMARK 1. Invoking Corollary 6, one can regard the Hilbert space $L^{2}(G / H, \mu)$ as a closed subspace of $L^{2}(G)$, that is the closed subspace consists of all $f \in L^{2}(G)$ which satisfies $R_{h} f=f$, for all $h \in H$. Then Theorem 2 and Proposition 4 guarantee that the linear map

$$
T_{H}: L^{2}(G) \rightarrow L^{2}(G / H, \mu) \subset L^{2}(G)
$$

is an orthogonal projection onto $L^{2}(G / H, \mu)$.

\section{Abstract convolution function algebras over homogeneous spaces of compact groups}

In this section, we present abstract structure of function $*$-algebras over homogeneous space (left coset spaces) of compact groups.

Let $G$ be a compact group and $H$ be a closed subgroup of $G$. Let,

$$
\mathcal{C}(G: H):=\left\{f \in \mathcal{C}(G): R_{h} f=f \forall h \in H\right\} .
$$

Then, one can also define

$$
A(G: H):=\left\{f \in \mathcal{C}(G): L_{h} f=f \forall h \in H\right\},
$$

and also

$$
A(G / H):=\left\{\varphi \in \mathcal{C}(G / H): L_{h} \varphi=\varphi \forall h \in H\right\} .
$$

For $1 \leq p<\infty$, one can define

$$
A^{p}(G: H):=\left\{f \in L^{p}(G): L_{h} f=f \forall h \in H\right\},
$$

and also

$$
A^{p}(G / H, \mu):=\left\{\varphi \in L^{p}(G / H, \mu): L_{h} \varphi=\varphi \forall h \in H\right\},
$$

where $L_{z} f(x):=f\left(z^{-1} x\right)$ and $R_{z} f(x):=f(x z)$, for $z, x \in G$.

It is easy to see that $A^{p}(G / H, \mu)$ is the topological closure of $A(G / H)$ in $L^{p}(G / H, \mu)$ and hence it is a closed linear subspace of $L^{p}(G / H, \mu)$. Also, one can readily check that $A^{p}(G: H)$ is the topological closure of $A(G: H)$ in $L^{p}(G)$ and hence it is a closed linear subspace of $L^{p}(G)$. 
The following remark presents our intuition to introduce the Banach function spaces $A^{p}(G / H, \mu)$.

REMARK 2. Let $G$ be a compact group and $H$ be a closed normal subgroup of $G$. Let $\mu$ be a $G$-invariant measure over the left coset space $G / H$ and $1 \leq p \leq \infty$. Let $\varphi \in \mathcal{C}(G / H)$ and $t \in H$. Then, for $x H \in G / H$, we have $t^{-1} x H=x H$. Hence, we can write

$$
L_{t} \varphi(x H)=\varphi\left(t^{-1} x H\right)=\varphi(x H) .
$$

Thus, we deduce that $\varphi \in A(G / H)$. Therefore, $A(G / H)=\mathcal{C}(G / H)$ and also $A^{p}(G / H, \mu)=L^{p}(G / H, \mu)$, if $H$ is normal in $G$.

We continue the paper by listing some basic observations.

Proposition 7. Let $G$ be a compact group and $H$ be a closed subgroup of $G$. Let $\mu$ be the normalized $G$-invariant measure on $G / H$ according to (4). Then

(1) $T_{H}$ maps $\mathcal{C}(G: H)$ onto $\mathcal{C}(G / H)$.

(2) $T_{H}$ maps $A(G: H)$ onto $A(G / H)$.

(3) $T_{H}$ maps $L^{p}(G: H)$ onto $A^{p}(G / H, \mu)$.

Proof. (1) It is straightforward.

(2) Let $f \in A(G: H), x \in G$ and $t \in H$. Then we have

$$
\begin{aligned}
L_{t} T_{H}(f)(x H) & =T_{H}(f)\left(t^{-1} x H\right) \\
& =\int_{H} f\left(t^{-1} x h\right) d h \\
& =\int_{H} f(x h) d h=T_{H}(f)(x H),
\end{aligned}
$$

which implies that $T_{H}(f) \in A(G / H)$. Let $\psi \in A(G / H)$. Then $\psi_{q} \in A(G: H)$ and $T_{H}\left(\psi_{q}\right)=\psi$. Hence, we deduce that $T_{H}$ maps $A(G: H)$ onto $A(G / H)$.

(3) Using (1) and since $A(G: H)$ is dense $L^{p}(G: H)$ and $A(G / H)$ is dense in $A^{p}(G / H)$ as well, we conclude that $T_{H}$ maps $L^{p}(G: H)$ onto $A^{p}(G / H, \mu)$.

Proposition 8. Let $G$ be a compact group and $H$ be a closed subgroup of $G$. Let $\mu$ be the normalized $G$-invariant measure on $G / H$ and $f, g \in L^{1}(G)$.

(1) For $x \in G$, we have

$$
T_{H}\left(f *_{G} g\right)(x H)=\int_{G / H}\left(\int_{H} f(y t)\left(\int_{H} g\left(t^{-1} y^{-1} x h\right) d h\right) d t\right) d \mu(y H) .
$$

(2) For $g \in A^{1}(G: H)$ and $x \in G$ we have

$$
T_{H}\left(f *_{G} g\right)(x H)=\int_{G / H} T_{H}(f)(y H) T_{H}(g)\left(y^{-1} x H\right) d \mu(y H) .
$$


Proof. (1) Let $f, g \in L^{1}(G)$ and $x \in G$. We can write

$$
\begin{aligned}
T_{H}\left(f *_{G} g\right)(x H) & =\int_{H} f *_{G} g(x h) d h \\
& =\int_{H}\left(\int_{G} f(y) g\left(y^{-1} x h\right) d y\right) d h .
\end{aligned}
$$

Then, using the Weil's formula, we get

$$
\begin{aligned}
T_{H}\left(f *_{G} g\right)(x H) & =\int_{H}\left(\int_{G} f(y) g\left(y^{-1} x h\right) d y\right) d h \\
& =\int_{H}\left(\int_{G / H}\left(\int_{H} f(y t) g\left((y t)^{-1} x h\right) d t\right) d \mu(y H)\right) d h \\
& =\int_{H}\left(\int_{G / H}\left(\int_{H} f(y t) g\left(t^{-1} y^{-1} x h\right) d t\right) d \mu(y H)\right) d h \\
& =\int_{G / H}\left(\int_{H} f(y t)\left(\int_{H} g\left(t^{-1} y^{-1} x h\right) d h\right) d t\right) d \mu(y H) .
\end{aligned}
$$

(2) Now suppose that $g \in A^{1}(G: H)$. Thus, $L_{t} g=g$ for all $t \in H$. Then, using (1) and the fact that $H$ is compact, we have

$$
\begin{aligned}
T_{H}\left(f *_{G} g\right)(x H) & =\int_{G / H}\left(\int_{H} f(y t)\left(\int_{H} g\left(t^{-1} y^{-1} x h\right) d h\right) d t\right) d \mu(y H) \\
& =\int_{G / H}\left(\int_{H} f(y t)\left(\int_{H} g\left(y^{-1} x h\right) d h\right) d t\right) d \mu(y H) \\
& =\int_{G / H}\left(\int_{H} f(y t) d t\right)\left(\int_{H} g\left(y^{-1} x h\right) d h\right) d \mu(y H) \\
& =\int_{G / H} T_{H}(f)(y H) T_{H}(g)\left(y^{-1} x H\right) d \mu(y H) .
\end{aligned}
$$

For $\psi \in \mathcal{C}(G / H)$, let $J \psi: G / H \rightarrow \mathbb{C}$ be given by

$$
J \psi(x H):=\int_{H} \psi(h x H) d h,
$$

for all $x H \in G / H$.

Then $J: \mathcal{C}(G / H) \rightarrow \mathcal{C}(G / H)$ given by $\psi \mapsto J \psi$ is a linear operator.

REMARK 3. Let $G$ be a compact group and $H$ be a closed normal subgroup of $G$. Then, for all $x \in G$ and $h \in H$, we have $h x H=x H$. Hence, for $\psi \in$ $\mathcal{C}(G / H)$ we get

$$
J \psi(x H)=\int_{H} \psi\left(h^{-1} x H\right) d h=\int_{H} \psi(x H) d h=\psi(x H) .
$$

Thus, we deduce that the linear operator $J: \mathcal{C}(G / H) \rightarrow \mathcal{C}(G / H)$ is the identity operator if $H$ is normal in $G$. 
The following theorem presents basic properties of the linear operator $J$ in the framework of abstract harmonic analysis.

TheOREM 9. Let $G$ be a compact group, $H$ be a closed subgroup of $G$, and $\mu$ be the normalized $G$-invariant measure over $G / H$.

(1) For each $1 \leq p<\infty$ and $\psi \in \mathcal{C}(G / H)$ we have

$$
\|J \psi\|_{L^{p}(G / H, \mu)} \leq\|\psi\|_{L^{p}(G / H, \mu)} .
$$

(2) $J$ maps $\mathcal{C}(G / H)$ onto $A(G / H)$.

Proof. (1) Let $1 \leq p<\infty$ and $\psi \in \mathcal{C}(G / H)$. Using compactness of $H$, we get

$$
\begin{aligned}
\|J \psi\|_{L^{p}(G / H, \mu)}^{p} & =\int_{G / H}|J \psi(x H)|^{p} d \mu(x H) \\
& =\int_{G / H}\left|\int_{H} \psi(h x H) d h\right|^{p} d \mu(x H) \\
& \leq \int_{G / H} \int_{H}|\psi(h x H)|^{p} d h d \mu(x H) .
\end{aligned}
$$

Again using compactness of $H$ and replacing $x$ by $h^{-1} x$ we get

$$
\begin{aligned}
\int_{G / H} \int_{H}|\psi(h x H)|^{p} d \mu(x H) d h & =\int_{H}\left(\int_{G / H}|\psi(h x H)|^{p} d \mu(x H)\right) d h \\
& =\int_{H}\left(\int_{G / H}|\psi(x H)|^{p} d \mu\left(h^{-1} x H\right)\right) d h \\
& =\int_{H}\left(\int_{G / H}|\psi(x H)|^{p} d \mu(x H)\right) d h \\
& =\|\psi\|_{L^{p}(G / H, \mu)}^{p} .
\end{aligned}
$$

(2) Let $\psi \in \mathcal{C}(G / H)$ and $t \in H$. Then we have

$$
\begin{aligned}
L_{t} J \psi(x H) & =J \psi\left(t^{-1} x H\right) \\
& =\int_{H} \psi\left(h t^{-1} x H\right) d h \\
& =\int_{H} \psi(h x H)=J \psi(x H),
\end{aligned}
$$

for all $x \in G$. This implies that $J \psi \in A(G / H)$. Now suppose that $\psi \in$ $A(G / H)$. Then we have

$$
J \psi(x H)=\int_{H} \psi(h x H) d h=\int_{H} \psi(x H) d h=\psi(x H),
$$

for all $x \in G$. Thus, $J \psi=\psi$. Hence, we deduce that $J$ maps $\mathcal{C}(G / H)$ onto $A(G / H)$. 
Then we deduce the following consequences.

Corollary 10. Let $G$ be a compact group and $H$ be a closed subgroup of $G$. Let $\mu$ be the normalized $G$-invariant measure on $G / H$ and $1 \leq p<\infty$.

(1) The linear operator $J: \mathcal{C}(G / H) \rightarrow A(G / H)$ has a unique extension to a bounded linear operator $J_{p}: L^{p}(G / H, \mu) \rightarrow A^{p}(G / H, \mu)$ which satisfies

$$
\left\|J_{p} \psi\right\|_{L^{p}(G / H, \mu)} \leq\|\psi\|_{L^{p}(G / H, \mu)}
$$

(2) The linear operator $J_{p}$ maps $L^{p}(G / H, \mu)$ onto $A^{p}(G / H, \mu)$.

REMARK 4. Let $G$ be a compact group and $H$ be a closed normal subgroup of $G$. Let $1 \leq p<\infty$. Then the extended the linear operator $J_{p}: L^{p}(G / H, \mu) \rightarrow A^{p}(G / H, \mu)$ is the identity operator.

Definition 11. Let $G$ be a compact group, $H$ be a closed subgroup of $G$, and $\mu$ be the normalized $G$-invariant measure over $G / H$. For $\varphi, \psi \in \mathcal{C}(G / H)$, let $\varphi *_{G / H} \psi: G / H \rightarrow \mathbb{C}$ be given by

$$
\varphi *_{G / H} \psi(x H)=\int_{G / H} \varphi(y H) J \psi\left(y^{-1} x H\right) d \mu(y H),
$$

for all $x H \in G / H$.

From now on, we call $\varphi *_{G / H} \psi$ as convolution of $\varphi$ and $\psi$. It is easy to check that the $\operatorname{map} *_{G / H}: \mathcal{C}(G / H) \times \mathcal{C}(G / H) \rightarrow \mathcal{C}(G / H)$ given by $(\varphi, \psi) \mapsto \varphi *_{G / H} \psi$ is bilinear. Also, it can be readily see that the linear space $\mathcal{C}(G / H)$ with respect to $*_{G / H}$ as multiplication is an algebra.

REMARK 5. Let $G$ be a compact group and $H$ be a closed normal subgroup of $G$. Also, let $\varphi, \psi \in \mathcal{C}(G / H)$ and $x \in G$. Invoking Remark 3, the linear map $J$ is the identity operator and hence we have

$$
\begin{aligned}
\varphi *_{G / H} \psi(x H) & =\int_{G / H} \varphi(y H) J \psi\left(y^{-1} x H\right) d \mu(y H) \\
& =\int_{G / H} \varphi(y H) \psi\left(y^{-1} x H\right) d \mu(y H) \\
& =\int_{G / H} \varphi(y H) \psi\left(y^{-1} H x H\right) d \mu(y H) \\
& =\int_{G / H} \varphi(y H) \psi\left((y H)^{-1} x H\right) d \mu(y H),
\end{aligned}
$$

for all $x H \in G / H$. Hence, we deduce that the convolution defined by (8) coincides with the canonical convolution over the quotient group $G / H$ if $H$ is normal in $G$, see [1], [23].

The following results state interesting properties of the convolution $*_{G / H}$. 
Proposition 12. Let $G$ be a compact group, $H$ be a closed subgroup of $G$, and $\mu$ be the normalized $G$-invariant measure over $G / H$. Also, let $\varphi, \psi \in$ $\mathcal{C}(G / H)$. Then we have

(1) $\varphi *_{G / H} \psi=T_{H}\left(\varphi_{q} *_{G} \psi_{q}\right)$.

(2) $\left(\varphi *_{G / H} \psi\right)_{q}=\varphi_{q} *_{G} \psi_{q}$.

(3) $L_{z}\left(\varphi *_{G / H} \psi\right)=\left(L_{z} \varphi\right) *_{G / H} \psi$.

Proof. (1) Let $x \in G$. Invoking definition of $*_{G}$ and since $H$ is compact, we can write

$$
\begin{aligned}
T_{H}\left(\varphi_{q} *_{G} \psi_{q}\right)(x H) & =\int_{H} \varphi_{q} *_{G} \psi_{q}(x h) d h \\
& =\int_{H}\left(\int_{G} \varphi_{q}(y) \psi_{q}\left(y^{-1} x h\right) d y\right) d h \\
& =\int_{H}\left(\int_{G} \varphi(y H) \psi\left(y^{-1} x h H\right) d y\right) d h \\
& =\int_{H}\left(\int_{G} \varphi(y H) \psi\left(y^{-1} x H\right) d y\right) d h \\
& =\int_{G} \varphi(y H) \psi\left(y^{-1} x H\right) d y .
\end{aligned}
$$

Then, using the Weil's formula, we get

$$
\begin{aligned}
T_{H}\left(\varphi_{q} *_{G} \psi_{q}\right)(x H) & =\int_{G} \varphi(y H) \psi\left(y^{-1} x H\right) d y \\
& =\int_{G / H}\left(\int_{H} \varphi(y h H) \psi\left((y h)^{-1} x H\right) d h\right) d \mu(y H) \\
& =\int_{G / H}\left(\int_{H} \varphi(y H) \psi\left(h^{-1} y^{-1} x H\right) d h\right) d \mu(y H) \\
& =\int_{G / H} \varphi(y H)\left(\int_{H} \psi\left(h^{-1} y^{-1} x H\right) d h\right) d \mu(y H) \\
& =\int_{G / H} \varphi(y H) J \psi\left(y^{-1} x H\right) d \mu(y H)=\varphi *_{G / H} \psi(x H) .
\end{aligned}
$$

(2) It is straightforward from (1).

(3) Let $z \in G$. Then we can write

$$
\begin{aligned}
L_{z}\left(\varphi *_{G / H} \psi\right)(x H) & =\varphi *_{G / H} \psi\left(z^{-1} x H\right) \\
& =\int_{G / H} \varphi(y H) J \psi\left(y^{-1} z^{-1} x H\right) d \mu(y H) \\
& =\int_{G / H} \varphi(y H) J \psi\left((z y)^{-1} x H\right) d \mu(y H) .
\end{aligned}
$$


Replacing $y$ by $z^{-1} y$ and using the fact that $\mu$ is $G$-invariant we get

$$
\begin{aligned}
\int_{G / H} \varphi(y H) J \psi\left((z y)^{-1} x H\right) d \mu(y H) \\
=\int_{G / H} \varphi\left(z^{-1} y H\right) J \psi\left(y^{-1} x H\right) d \mu\left(z^{-1} y H\right) \\
=\int_{G / H} \varphi\left(z^{-1} y H\right) J \psi\left(y^{-1} x H\right) d \mu(y H) \\
=\int_{G / H} L_{z} \varphi(y H) J \psi\left(y^{-1} x H\right) d \mu(y H) \\
=\left(L_{z} \varphi\right) *{ }_{G / H} \psi(x H) .
\end{aligned}
$$

Proposition 13. Let $G$ be a compact group, $H$ be a closed subgroup of $G$, and $\mu$ be the normalized $G$-invariant measure over $G / H$. Also, let $f, g \in \mathcal{C}(G)$ with $f \in \mathcal{C}(G: H)$. Then we have

$$
T_{H}\left(f *_{G} g\right)=T_{H}(f) *_{G / H} T_{H}(g) .
$$

In particular, for all $\varphi \in \mathcal{C}(G / H)$ and $g \in \mathcal{C}(G)$, we have

$$
T_{H}\left(\varphi_{q} *_{G} g\right)=\varphi *_{G / H} T_{H}(g) .
$$

Proof. Let $f, g \in \mathcal{C}(G)$ with $f \in \mathcal{C}(G: H)$. Then using Proposition 8, for $x \in G$, we get

$$
\begin{aligned}
T_{H}\left(f *_{G} g\right)(x H) & =\int_{G / H}\left(\int_{H} f(y t)\left(\int_{H} g\left(t^{-1} y^{-1} x h\right) d h\right) d t\right) d \mu(y H) \\
& =\int_{G / H} f(y)\left(\int_{H}\left(\int_{H} g\left(t^{-1} y^{-1} x h\right) d h\right) d t\right) d \mu(y H) \\
& =\int_{G / H} T_{H}(f)(y H)\left(\int_{H}\left(\int_{H} g\left(t^{-1} y^{-1} x h\right) d h\right) d t\right) d \mu(y H) \\
& =\int_{G / H} T_{H}(f)(y H)\left(\int_{H} T_{H}(g)\left(t^{-1} y^{-1} x H\right) d t\right) d \mu(y H) \\
& =\int_{G / H} T_{H}(f)(y H) J\left(T_{H}(g)\right)\left(y^{-1} x H\right) d \mu(y H) \\
& =T_{H}(f) *_{G / H} T_{H}(g)(x H) .
\end{aligned}
$$

Now let $\varphi \in \mathcal{C}(G / H)$. Then $f:=\varphi_{q} \in \mathcal{C}(G: H)$. Thus, we get

$$
T_{H}\left(\varphi_{q} *_{G} g\right)=T_{H}\left(\varphi_{q}\right) *_{G / H} T_{H}(g)=\varphi *_{G / H} T_{H}(g) .
$$

REMARK 6. Let $H$ be a closed normal subgroup of a compact group $G$. Let $\mu$ be the normalized $G$-invariant measure over $G / H$. Then $\mu$ is a Haar 
measure over the quotient group $G / H$. Then using Proposition 8, for $x \in G$ and $f, g \in \mathcal{C}(G)$, we can write

$$
\begin{aligned}
T_{H}\left(f *_{G} g\right)(x H) & =\int_{G / H}\left(\int_{H} f(y t)\left(\int_{H} g\left(t^{-1} y^{-1} x h\right) d h\right) d t\right) d \mu(y H) \\
& =\int_{G / H}\left(\int_{H} f(y t) d t\right)\left(\int_{H} g\left(y^{-1} x h\right) d h\right) d \mu(y H) \\
& =\int_{G / H} T_{H}(f)(x H) T_{H}(g)\left(y^{-1} x H\right) d \mu(y H) \\
& =T_{H}(f) *_{G / H} T_{H}(g) .
\end{aligned}
$$

This property of convolution over quotient groups have been appeared in [23] as well.

The next result shows that the algebra $\mathcal{C}(G / H)$ with respect to the norm $\|\cdot\|_{L^{p}(G / H, \mu)}$ is a normed algebra, for all $1 \leq p<\infty$.

TheOREm 14. Let $G$ be a compact group and $H$ be a closed subgroup of $G$. Let $\mu$ be the normalized $G$-invariant measure on $G / H$ and $1 \leq p<\infty$. Then, for all $\varphi, \psi \in \mathcal{C}(G / H)$, we have

$$
\left\|\varphi *_{G / H} \psi\right\|_{L^{p}(G / H, \mu)} \leq\|\varphi\|_{L^{p}(G / H, \mu)}\|\psi\|_{L^{p}(G / H, \mu)} .
$$

Proof. Let $\varphi, \psi \in \mathcal{C}(G / H)$ and $1 \leq p<\infty$. Then, using (1), (6), and Proposition 12 , we have

$$
\begin{aligned}
\left\|\varphi *_{G / H} \psi\right\|_{L^{p}(G / H, \mu)} & =\left\|\left(\varphi *_{G / H} \psi\right)_{q}\right\|_{L^{p}(G)} \\
& =\left\|\varphi_{q} *_{G} \psi_{q}\right\|_{L^{p}(G)} \\
& \leq\left\|\varphi_{q}\right\|_{L^{p}(G)}\left\|\psi_{q}\right\|_{L^{p}(G)} \\
& =\|\varphi\|_{L^{p}(G / H, \mu)}\|\psi\|_{L^{p}(G / H, \mu)} .
\end{aligned}
$$

Then we can present the following interesting result.

THEOREM 15. Let $G$ be a compact group and $H$ be a closed subgroup of $G$. Let $\mu$ be the normalized $G$-invariant measure on $G / H$ and also $1 \leq p<\infty$. The convolution map $*_{G / H}: \mathcal{C}(G / H) \times \mathcal{C}(G / H) \rightarrow \mathcal{C}(G / H)$ given by $(8)$ has a unique extension to $*_{G / H}^{p}: L^{p}(G / H, \mu) \times L^{p}(G / H, \mu) \rightarrow L^{p}(G / H, \mu)$, in which the Banach function space $L^{p}(G / H, \mu)$ equipped with the extended convolution is a Banach algebra.

Proof. Let $\varphi, \psi \in L^{p}(G / H, \mu)$. Invoking density of $\mathcal{C}(G / H)$ in $L^{p}(G / H, \mu)$, let $\left\{\varphi_{n}\right\},\left\{\psi_{n}\right\} \in \mathcal{C}(G / H)$ with $\varphi=\lim _{n} \varphi_{n}$ and $\psi=\lim _{n} \psi_{n}$. Then we define

$$
\varphi *_{G / H}^{p} \psi:=\lim _{n} \varphi_{n} *_{G / H} \psi_{n} .
$$


Then $*_{G / H}^{p}: L^{p}(G / H, \mu) \times L^{p}(G / H, \mu) \rightarrow L^{p}(G / H, \mu)$ is well-defined and satisfies

$$
\left\|\varphi *_{G / H} \psi\right\|_{L^{p}(G / H, \mu)} \leq\|\varphi\|_{L^{p}(G / H, \mu)}\|\psi\|_{L^{p}(G / H, \mu)} .
$$

This equivalently implies that the Banach function space $L^{p}(G / H, \mu)$ equipped with the extended convolution is a Banach algebra.

Also, we deduce the following corollary concerning explicit construction of $*_{G / H}^{p}$.

Corollary 16. Let $G$ be a compact group and $H$ be a closed subgroup of $G$. Let $\mu$ be the normalized $G$-invariant measure on $G / H$ and also $1 \leq p<\infty$. Then, for all $\varphi, \psi \in L^{p}(G / H, \mu)$, we have

$$
\varphi *_{G / H}^{p} \psi(x H)=\int_{G / H} \varphi(y H) J_{p} \psi\left(y^{-1} x H\right) d \mu(y H),
$$

for $x H \in G / H$.

We then present the following corollary concerning the Banach function subspaces $A^{p}(G / H, \mu)$.

Corollary 17. Let $G$ be a compact group and $H$ be a closed subgroup of $G$. Let $\mu$ be the normalized $G$-invariant measure on $G / H$ and also $1 \leq p<\infty$. Then $A^{p}(G / H, \mu)$ is a Banach function sub-algebra of $L^{p}(G / H, \mu)$.

REMARK 7. Let $G$ be a compact group and $H$ be a closed normal subgroup of $G$. Let $\mu$ be the normalized $G$-invariant measure on $G / H$ and also $1 \leq$ $p<\infty$. Then automatically $\mu$ is precisely a Haar measure of the compact quotient group $G / H$. Also, let $\varphi, \psi \in L^{p}(G / H, \mu)$ and $x \in G$. Invoking Remark 4 , the linear map $J_{p}$ is the identity operator and hence we have

$$
\begin{aligned}
\varphi *_{G / H}^{p} \psi(x H) & =\int_{G / H} \varphi(y H) J_{p} \psi\left(y^{-1} x H\right) d \mu(y H) \\
& =\int_{G / H} \varphi(y H) \psi\left(y^{-1} x H\right) d \mu(y H) \\
& =\int_{G / H} \varphi(y H) \psi\left(y^{-1} H x H\right) d \mu(y H),
\end{aligned}
$$

for $x H \in G / H$. Hence, we deduce that the extended convolution $*_{G / H}^{p}$ coincides with the canonical convolution over the quotient group $G / H$ if $H$ is normal in $G$.

Definition 18. Let $G$ be a compact group and $H$ be a closed subgroup of $G$. For $\varphi \in \mathcal{C}(G / H)$, let $\varphi^{*} G / H: G / H \rightarrow \mathbb{C}$ be given by

$$
\varphi^{* G / H}(x H)=\int_{H} \overline{\varphi\left(h^{-1} x^{-1} H\right)} d h,
$$

for all $x H \in G / H$. 
Let $x H=y H$, for $x, y \in G$. Then we have $y=x t$ for some $t \in H$. Hence, we can write

$$
\int_{H} \overline{\varphi\left(h^{-1} x^{-1} H\right)} d h=\int_{H} \overline{\varphi\left(h^{-1} t^{-1} x^{-1} H\right)} d h=\int_{H} \overline{\varphi\left(h^{-1} y^{-1} H\right)} d h,
$$

which implies that $\varphi^{*_{G / H}}(x H)=\varphi^{*_{G / H}}(y H)$. This guarantees that $\varphi^{*_{G / H}}$ is a well-defined function over $G / H$.

From now on, we call $\varphi^{* G / H}$ as involution of $\varphi$. It is easy to check that the map ${ }^{*_{G / H}}: \mathcal{C}(G / H) \rightarrow \mathcal{C}(G / H)$ given by $\varphi \mapsto \varphi^{*_{G / H}}$ is conjugate linear.

REMARK 8. Let $G$ be a compact group and $H$ be a closed normal subgroup of $G$. Also, let $\varphi \in \mathcal{C}(G / H)$ and $x \in G$. Then, for all $x \in G$ and $h \in H$, we have $h x H=x H$. Hence, for $\psi \in \mathcal{C}(G / H)$ we get

$$
\varphi^{* / H}(x H)=\int_{H} \overline{\varphi\left(h^{-1} x^{-1} H\right)} d h=\int_{H} \overline{\varphi\left(x^{-1} H\right)} d h=\varphi\left(x^{-1} H\right) .
$$

Thus, we deduce that the involution defined by (9) coincides with the canonical involution over the compact quotient group $G / H$ if $H$ is normal in $G$.

Proposition 19. Let $G$ be a compact group and $H$ be a closed subgroup of $G$. Let $\varphi \in \mathcal{C}(G / H), 1 \leq p<\infty$, and $\mu$ be the normalized $G$-invariant measure on $G / H$. Then we have

(1) $\varphi^{*^{G / H} *^{G / H}}=J \varphi$.

(2) $\varphi^{*_{G / H}}=T_{H}\left(\varphi_{q}^{*_{G}}\right)$.

(3) $\left\|\varphi^{* G / H}\right\|_{L^{p}(G / H, \mu)} \leq\|\varphi\|_{L^{p}(G / H, \mu)}$.

Proof. (1) Let $x \in G$ and $h \in H$. Then we have

$$
\overline{\varphi^{* G / H}\left(h^{-1} x^{-1} H\right)}=\int_{H} \varphi\left(t^{-1} x h H\right) d t=\int_{H} \varphi\left(t^{-1} x H\right) d t .
$$

Thus, we get

$$
\varphi^{*^{G / H} *^{G / H}}(x H)=\int_{H} \overline{\varphi^{* G / H}\left(h^{-1} x^{-1} H\right)} d h=\int_{H} \varphi\left(t^{-1} x H\right) d t=J \varphi(x H) .
$$

(2) Let $x \in G$. Then we have

$$
\begin{aligned}
T_{H}\left(\varphi_{q}^{*_{G}}\right)(x H) & =\int_{H} \varphi_{q}^{*_{G}}(x h) d h \\
& =\int_{H} \overline{\varphi_{q}\left(h^{-1} x^{-1}\right)} d h=\int_{H} \overline{\varphi\left(h^{-1} x^{-1} H\right)} d h=\varphi^{*^{G} H}(x H) .
\end{aligned}
$$

(3) Using (1), compactness of $H$, and also the Weil's formula we have

$$
\begin{aligned}
\left\|\varphi^{* G / H}\right\|_{L^{p}(G / H, \mu)} & =\int_{G / H}\left|\varphi^{* G / H}(x H)\right|^{p} d \mu(x H) \\
& =\int_{G / H}\left|T_{H}\left(\varphi_{q}^{* G}\right)(x H)\right|^{p} d \mu(x H)
\end{aligned}
$$




$$
\begin{aligned}
& \leq \int_{G / H} T_{H}\left(\left|\varphi_{q}^{*_{G}}\right|^{p}\right)(x H) d \mu(x H) \\
& =\int_{G}\left|\varphi_{q}^{*_{G}}(x)\right|^{p} d x=\left\|\varphi_{q}\right\|_{L^{p}(G)}=\|\varphi\|_{L^{p}(G / H, \mu)} .
\end{aligned}
$$

Corollary 20. Let $G$ be a compact group and $H$ be a closed subgroup of $G$. Let $\varphi \in A(G / H), 1 \leq p<\infty$, and $\mu$ be the normalized G-invariant measure on $G / H$. Then we have

(1) $\varphi^{*^{G / H} *^{G / H}}=\varphi$.

(2) $\left\|\varphi^{* G / H}\right\|_{L^{p}(G / H, \mu)}=\|\varphi\|_{L^{p}(G / H, \mu)}$.

(3) $\left(\varphi^{*} G / H\right)_{q}=\varphi_{q}^{* G}$.

Then we can deduce the following result.

Proposition 21. Let $G$ be a compact group and $H$ be a closed subgroup of $G$. Also, let $\varphi, \psi \in \mathcal{C}(G / H)$. Then we have

$$
\left(\varphi *_{G / H} \psi\right)^{*^{G / H}}=\psi^{*^{G / H}} *_{G / H} \varphi^{*^{G / H}} .
$$

Proof. Using Propositions 12, 19, and also (2) we have

$$
\begin{aligned}
\left(\varphi *_{G / H} \psi\right)^{*^{G / H}} & =T_{H}\left(\left(\varphi *_{G / H} \psi\right)_{q}^{*^{G}}\right) \\
& =T_{H}\left(\left(\varphi_{q} *_{G} \psi_{q}\right)^{*^{G}}\right)=T_{H}\left(\psi_{q}^{*^{G}} *_{G} \varphi_{q}^{*^{G}}\right) .
\end{aligned}
$$

Since $\varphi_{q}^{*^{G}} \in A(G: H)$, using Proposition 8 we can write

$$
\begin{aligned}
T_{H}\left(\psi_{q}^{*^{G}} *_{G} \varphi_{q}^{*^{G}}\right)(x H) & =\int_{G / H} T_{H}\left(\psi_{q}^{*^{G}}\right)(y H) T_{H}\left(\varphi_{q}^{*^{G}}\right)\left(y^{-1} x H\right) d \mu(y H) \\
& =\int_{G / H} T_{H}\left(\psi_{q}^{*^{G}}\right)(y H) J T_{H}\left(\varphi_{q}^{*^{G}}\right)\left(y^{-1} x H\right) d \mu(y H) \\
& =T_{H}\left({\psi_{q}^{*}}^{*^{G}}\right) *_{G / H} T_{H}\left(\varphi_{q}^{*^{G}}\right)(x H) \\
& =\psi^{*^{G / H}} *_{G / H} \varphi^{*^{G / H}}(x H),
\end{aligned}
$$

for $x \in G$, which completes the proof.

Then we can summarize our recent results as follows.

Corollary 22. Let $G$ be a compact group and $H$ be a closed subgroup of $G$. The linear space $A(G / H)$ equipped with the convolution $*_{G / H}$ and the involution $*_{G / H}$ is a normed $*$-algebra.

The following proposition presents properties of involution over $L^{p}$-spaces.

Proposition 23. Let $G$ be a compact group and $H$ be a closed subgroup of $G$. Let $\mu$ be the normalized $G$-invariant measure on $G / H$ and also $1 \leq$ $p<\infty$. The involution map ${ }^{*^{G / H}}: \mathcal{C}(G / H) \rightarrow \mathcal{C}(G / H)$ given by (9) has 
a unique extension to ${ }^{*^{G / H}}: L^{p}(G / H, \mu) \rightarrow L^{p}(G / H, \mu)$ which, for all $\varphi \in$ $L^{p}(G / H, \mu)$, satisfies

(1) $\varphi^{*^{G / H} *^{G / H}}=J_{p} \varphi$.

(2) $\varphi^{*_{G / H}}=T_{H}\left(\varphi_{q}^{*_{G}}\right)$.

(3) $\left\|\varphi^{* G / H}\right\|_{L^{p}(G / H, \mu)} \leq\|\varphi\|_{L^{p}(G / H, \mu)}$.

Proof. Let $\varphi \in L^{p}(G / H, \mu)$. Invoking density of $\mathcal{C}(G / H)$ in $L^{p}(G / H, \mu)$, let $\left\{\varphi_{n}\right\} \in \mathcal{C}(G / H)$ with $\varphi=\lim _{n} \varphi_{n}$. Then we define

$$
\varphi^{*^{G / H}}:=\lim _{n} \varphi_{n}^{*^{G / H}} .
$$

Then ${ }^{*^{G / H}}: L^{p}(G / H, \mu) \rightarrow L^{p}(G / H, \mu)$ is well-defined and satisfies (1)-(3).

Corollary 24. Let $G$ be a compact group and $H$ be a closed subgroup of $G$. Let $\mu$ be a $G$-invariant measure on $G / H$ and also $1 \leq p<\infty$. Then we have

for $x \in G$.

$$
\varphi^{* G / H}(x H)=\int_{H} \overline{\varphi\left(h^{-1} x^{-1} H\right)} d h,
$$

Corollary 25. Let $G$ be a compact group, $H$ be a closed subgroup of $G$, and $\mu$ be the normalized $G$-invariant measure on $G / H$. Let $\varphi \in A^{p}(G / H, \mu)$ with $1 \leq p<\infty$. Then we have

(1) $\varphi^{*^{G / H} *^{G / H}}=\varphi$.

(2) $\left\|\varphi^{* G / H}\right\|_{L^{p}(G / H, \mu)}=\|\varphi\|_{L^{p}(G / H, \mu)}$.

(3) $\left(\varphi^{* G / H}\right)_{q}=\varphi_{q}^{* G}$.

The next result summarizes our recent results in terms of Banach *algebras.

THEOREM 26. Let $G$ be a compact group and $H$ be a closed subgroup of $G$. Let $\mu$ be the normalized $G$-invariant measure on $G / H$ and also $1 \leq p<\infty$. The Banach function algebra $A^{p}(G / H, \mu)$ equipped with the extended involution is a Banach function *-algebra.

Acknowledgment. The author would like to express his deepest gratitude to Prof. Hans G. Feichtinger for his valuable comments.

\section{REFERENCES}

[1] A. Derighetti, On the multipliers of a quotient group, Bull. Sci. Math. (2) 107 (1983), no. 1, 3-23. MR 0699988

[2] A. Derighetti, Convolution operators on groups, Lecture Notes of the Unione Matematica Italiana, vol. 11, Springer, Heidelberg, UMI, Bologna, 2011. MR 2809956

[3] J. Dixmier, $C^{*}$-Algebras, North-Holland Publishing Company, Amsterdam, 1977.

[4] H. G. Feichtinger, On a class of convolution algebras of functions, Ann. Inst. Fourier (Grenoble) 27 (1977), 135-162. MR 0470610 
[5] H. G. Feichtinger, Banach convolution algebras of functions II, Monatsh. Math. 87 (1979), no. 3, 181-207. MR 0536089

[6] H. G. Feichtinger, On a new Segal algebra, Monatsh. Math. 92 (1981), no. 4, 269-289. MR 0643206

[7] H. G. Feichtinger and K. H. Gröchenig, Banach spaces related to integrable group representations and their atomic decompositions. I, J. Funct. Anal. 86 (1989), no. 2, 307-340. MR 1021139

[8] H. G. Feichtinger and K. H. Gröchenig, Banach spaces related to integrable group representations and their atomic decompositions. II, Monatsh. Math. 108 (1989), no. 2-3, 129-148.

[9] G. B. Folland, A course in abstract harmonic analysis, CRC Press, Boca Ratton, FL, 1995.

[10] A. Ghaani Farashahi, Abstract non-commutative harmonic analysis of coherent state transforms, Ph.D. thesis, Ferdowsi University of Mashhad (FUM), Mashhad, 2012.

[11] A. Ghaani Farashahi, Convolution and involution on function spaces of homogeneous spaces, Bull. Malays. Math. Sci. Soc. (2) 36 (2013), no. 4, 1109-1122. MR 3108799

[12] A. Ghaani Farashahi, Abstract relative Fourier transforms over canonical homogeneous spaces of semi-direct product groups with Abelian normal factor, J. Korean Math. Soc. (2016). DOI:10.4134/JKMS.j150610.

[13] A. Ghaani Farashahi, Abstract harmonic analysis of relative convolutions over canonical homogeneous spaces of semidirect product groups, J. Aust. Math. Soc. 101 (2016), no. 2, 171-187. MR 3569063

[14] A. Ghaani Farashahi, Abstract Plancherel (trace) formulas over homogeneous spaces of compact groups, Canad. Math. Bull. 60 (2017), 111-121.

[15] E. Hewitt and K. A. Ross, Absrtact harmonic analysis, vol. 1, Springer-Verlag, New York, 1963.

[16] E. Hewitt and K. A. Ross, Absrtact harmonic analysis, vol. 2, Springer-Verlag, Berlin, 1970.

[17] V. Kisil, Relative convolutions. I. Properties and applications, Adv. Math. 147 (1999), no. $1,35-73$. MR 1725814

[18] V. Kisil, Erlangen program at large: An overview, Advances in applied analysis, Trends Math., Birkhäuser/Springer Basel AG, Basel, 2012, pp. 1-94.

[19] V. Kisil, Geometry of Möbius transformations. Elliptic, parabolic and hyperbolic actions of $S L_{2}(\mathbb{R})$, Imperial College Press, London, 2012.

[20] V. Kisil, Operator covariant transform and local principle, J. Phys. A 45 (2012), no. 24, 244022.

[21] V. Kisil, Calculus of operators: Covariant transform and relative convolutions, Banach J. Math. Anal. 8 (2014), no. 2, 156-184.

[22] G. J. Murphy, $C^{*}$-algebras and operator theory, Academic Press, Inc., Boston, MA, 1990. MR 1074574

[23] H. Reiter and J. D. Stegeman, Classical harmonic analysis and locally compact groups, 2nd ed., London Mathematical Society Monographs. New Series, vol. 22, The Clarendon Press, Oxford University Press, New York, 2000. MR 1802924

Arash Ghaani Farashahi, Numerical Harmonic Analysis Group (NuHaG), Faculty of Mathematics, University of Vienna, Oskar-Morgenstern-Platz 1, A-1090

Vienna, Austria

E-mail address: arash.ghaani.farashahi@univie.ac.at;

ghaanifarashahi@hotmail.com 